\title{
A Hopf Bifurcation in a Three-Component Reaction-Diffusion System with a Chemoattraction
}

\author{
YoonMee Ham, ${ }^{1}$ Sang-Gu Lee, ${ }^{2}$ and Quoc Phong Vu ${ }^{3,4}$ \\ ${ }^{1}$ Department of Mathematics, Kyonggi University, Suwon 443-760, Republic of Korea \\ ${ }^{2}$ Department of Mathematics, Sungkyunkwan University, Suwon 440-746, Republic of Korea \\ ${ }^{3}$ Department of Mathematics, Ohio University, Athens, OH 45701, USA \\ ${ }^{4}$ Vietnam Institute of Advanced Study in Mathematics, Hanoi, Vietnam \\ Correspondence should be addressed to Sang-Gu Lee; sglee@skku.edu
}

Received 21 August 2013; Accepted 23 October 2013

Academic Editor: Abdelghani Bellouquid

Copyright (C) 2013 YoonMee Ham et al. This is an open access article distributed under the Creative Commons Attribution License, which permits unrestricted use, distribution, and reproduction in any medium, provided the original work is properly cited.

We consider a three-component reaction-diffusion system with a chemoattraction. The purpose of this work is to analyze the chemotactic effects due to the gradient of the chemotactic sensitivity and the shape of the interface. Conditions for existence of stationary solutions and the Hopf bifurcation in the interfacial problem as the bifurcation parameters vary are obtained analytically.

\section{Introduction}

We are interested in the effects of diffusivity and chemotaxis on the competition of several species for limited resources. Chemotaxis is an oriented movement of cells in response to a concentration gradient of chemical substances in their environment. It was observed that diffusivity and chemotaxis of cells play a dominant role in cell growth; when several species of cells compete for limited resources, the species with a smaller diffusion rate and larger chemotaxis rate grow better, even when other species have superior growth kinetics.

Mathematical modeling on chemotaxis was initiated in 1970 by Keller and Segel (see [1]) with the use of the following system of PDEs:

$$
\begin{gathered}
u_{t}=\nabla \cdot(\nabla u-\chi u \nabla v), \\
v_{t}=D \nabla^{2} v+a u-b v, \quad t>0, \quad \mathbf{x} \in \mathbb{R}^{n},
\end{gathered}
$$

where $D$ is a diffusion coefficient, $a$ and $b$ are positive constants, and $\chi$ is the chemotaxis coefficient. In many biological processes, cells often interact with combinations of repulsive and attractive signalling chemicals to produce various interesting biological patterns. In this paper, we consider the attraction chemotaxis system [2-4]:

$$
\begin{gathered}
\varepsilon \sigma u_{t}=\varepsilon^{2} \nabla^{2} u-\varepsilon \kappa \nabla(u \cdot \nabla \chi(v))-u+H(u-a(w))-v, \\
v_{t}=\nabla^{2} v+\mu u-v, \quad t>0, \mathbf{x} \in \mathbb{R}^{n}, \\
w_{t}=\nabla^{2} w+u+v-w-s_{0}, \quad t>0, \mathbf{x} \in \mathbb{R}^{n},
\end{gathered}
$$

where $\varepsilon, \sigma, \mu$, and $s_{0}$ are positive constants, $H$ is a Heaviside step function, and $a^{\prime}(w)>0$ for all $w$. Here, $\nabla$ is the gradient operator, $\chi$ is the chemical sensitivity function of the chemical repulsion satisfying $\chi^{\prime}(v) \geq 0$ for $v>0$, and $\kappa$ is a positive constant.

Chemotaxis describes the direct migration of cells along the concentration gradient of a specific chemical produced by the cells. The prototype of the population-based chemotaxis model was described in the above mentioned work of Keller and Segal [1].

Schaaf [5] discussed the existence of nonconstant equilibrium solutions which exhibit aggregating patterns in a bounded domain. In $[4,6]$, equations describing the dynamics of the interfaces near equilibrium and the stability of the planar standing pulse solutions in the channel domain are 
obtained for sufficiently small $\varepsilon$. Results for several versions of the Keller-Segel system and its related models are discussed in Horstmann [7, 8] and Ward [9]. The effect of chemotaxis or that of lateral inhibition on an activator in reaction-diffusion systems has been studied by several authors (see [10-13]).

In the present work, chemotaxis growth under the influence of lateral inhibition in a three-component reactiondiffusion system is considered. We derive a free boundary problem of this system when $\varepsilon=0$ and then find conditions which are necessary for occurrence of the Hopf bifurcation of chemotaxis and the lateral inhibition on an activator. We derive an evolutional equation of interfaces that is controlled by the two inhibitors $v$ and $w$.

Suppose that there is only one interfacial curve $x=\eta(t)$ in $[0, \infty)$ in such a way that $[0, \infty)=\Omega_{1} \cup \eta(t) \cup \Omega_{0}$, where $\Omega_{1}=$ $\{x \in[0, \infty): u(x, t)>a(w(x, t))\}$ and $\Omega_{0}=\{x \in[0, \infty):$ $u(x, t)<a(w(x, t))\}$. Let $\left(x_{0}, t_{0}\right)$ lie on this curve; that is, $x_{0}=$ $\eta\left(t_{0}\right)$. Using a stretching transformation at $\left(x_{0}, t_{0}\right)$ we make the following substitutions:

$$
\xi=\frac{x-x_{0}}{\varepsilon}, \quad \rho=\frac{t-t_{0}}{\varepsilon} .
$$

Then, the system $(2)$ at $\left(x_{0}, t_{0}\right)$ becomes

$$
\begin{gathered}
\sigma u_{\rho}=u_{\xi \xi}-\kappa \chi^{\prime}\left(v_{0}\right) v_{x} u_{\xi}+F\left(u, v_{0}, w_{0}\right), \\
F(u, v, w)=-u+H(u-a(w))-v
\end{gathered}
$$

and the boundary conditions are

$$
u( \pm \infty)=h_{ \pm}\left(v_{0}\right)
$$

when $\varepsilon$ tends to zero, where $v_{0}=v\left(x_{0}, t_{0}\right)$ and $w_{0}=w\left(x_{0}, t_{0}\right)$. We put the equation into a traveling coordinate system by setting $z=\xi-\theta \rho$ with velocity $\theta$. Thus, $U(z)=u(\xi, \rho)$ satisfies the following conditions:

$$
\begin{gathered}
U_{z z}+\left(\sigma \theta-\kappa \chi^{\prime}\left(v_{0}\right) v_{x}\right) U_{z}+F\left(U, v_{0}, w_{0}\right)=0 \\
U( \pm \infty)=h_{ \pm}\left(v_{0}\right)
\end{gathered}
$$

The existence of a solution $U(z)$ is given in $[12,14]$ and $\theta$ satisfies $\sigma \theta=C\left(v_{0}\right)+\kappa \chi^{\prime}\left(v_{0}\right) v_{x}\left(x_{0}, t_{0}\right)$. Hence, the velocity of the one-dimensional interface $\eta(t)$ is given by

$$
\frac{d \eta(t)}{d t}=\frac{1}{\sigma}\left(C\left(v_{i}\right)+\kappa \chi^{\prime}\left(v_{i}\right) v_{x}(\eta(t), t)\right), \quad x \in \eta(t),
$$

where $v_{i}$ is the value of $v$ on the interface $\eta(t)$ and $C$ is a continuously differentiable function defined on an interval $I:=(-a(w), 1-a(w))$, which is given by [14-16]

$$
\begin{aligned}
& C(v(\eta) ; a(w(\eta))) \\
& \quad=-\frac{1-2 a(w(\eta))-2 v(\eta)}{\sqrt{(v(\eta)+a(w))(1-a(w(\eta))-v(\eta))}} .
\end{aligned}
$$

Hence, a free boundary problem of (2) when $\varepsilon$ is equal to zero is given by

$$
\begin{gathered}
v_{t}=\nabla^{2} v-(\mu+1) v+\mu, \quad t>0, x \in \Omega_{1}, \\
v_{t}=\nabla^{2} v-(\mu+1) v, \quad t>0, x \in \Omega_{0}, \\
v(\eta(t)-0, t)=v(\eta(t)+0, t), \\
v_{x}(\eta(t)-0, t)=v_{x}(\eta(t)+0, t), \\
\lim _{x \rightarrow \infty} v(x, t)=0, \\
w_{t}=\nabla^{2} w-w+1-s_{0}, \quad t>0, x \in \Omega_{1}, \\
w_{t}=\nabla^{2} w-w-s_{0}, \quad t>0, x \in \Omega_{0}, \\
w(\eta(t)-0, t)=w(\eta(t)+0, t), \\
w_{x}(\eta(t)-0, t)=w_{x}(\eta(t)+0, t), \\
\lim _{x \rightarrow \infty} w(x, t)=-s_{0} .
\end{gathered}
$$

In this paper, we establish the existence of the Hopf bifurcation described above by an application of the implicit function theorem along the lines of the results in [17]. In order to apply the implicit function theorem, we require more regularity of the solution than that obtained in the papers $[4,6,13]$. Our approach to the problem of well-posedness and to the Hopf bifurcation is to write (9) in the form of an abstract evolution equation on a Banach space, which is the product of a function space and an interval of real numbers. Once we have done this, we are able to apply standard results from the theory of nonlinear evolution equations (see for instance, [18]) to show the well-posedness of the problem and, more importantly, to give an analysis of the Hopf bifurcation.

The organization of the paper is as follows. In Section 2, a change of variables is given which regularizes problem (9) in such a way that results from the theory of nonlinear evolution equations can be applied. In this way, we obtain a regularity of the solution which is sufficient for an analysis of the bifurcation. In Section 3, we show the existence of equilibrium solutions for (9) and obtain the linearization of problem (9). In the last section, we investigate the conditions to obtain the periodic solutions and the bifurcation of the interface problem as the parameter $\sigma$ varies.

\section{Regularization of the Interface Equation}

Now, we consider the existence problem of (9):

$$
\begin{gathered}
v_{t}=\frac{\partial^{2} v}{\partial x^{2}}-(\mu+1) v+\mu H(x-\eta(t)), \\
w_{t}=\frac{\partial^{2} w}{\partial x^{2}}-w+H(x-\eta(t))-s_{0}, \\
v_{x}(0, t)=0, \quad \lim _{x \rightarrow \infty} v_{x}(x, t)=0, \quad t>0, \\
w_{x}(0, t)=0, \quad \lim _{x \rightarrow \infty} w_{x}(x, t)=-s_{0}, \quad t>0,
\end{gathered}
$$




$$
\begin{aligned}
& \sigma \eta^{\prime}(t)= C(v(\eta) ; a(w(\eta))) \\
&+\kappa \chi^{\prime}(v(\eta(t), t)) v_{x}(\eta(t), t), \\
& t>0 ; \quad \eta(0)=\eta_{0} .
\end{aligned}
$$

Let $\widehat{w}(x, t)=w(x, t)+s_{0}$. Let $A$ be an operator defined by $A:=-\left(d^{2} / d x^{2}\right)+\mu+1$ with domain $D(A)=\left\{v \in H^{2,2}(\mathbb{R})\right.$ : $\left.v_{x}(0, t)=0, \lim _{x \rightarrow \infty} v_{x}(x, t)=0\right\}$. Let $A_{0}:=-\left(\partial^{2} / \partial x^{2}\right)+1$ with domain $D\left(A_{0}\right)=\left\{w \in H^{2,2}((0, \infty)): \widehat{w}_{x}(0, t)=\right.$ $\left.0, \lim _{x \rightarrow \infty} \widehat{w}_{x}(x, t)=0\right\}$. In order to apply semigroup theory to $(10)$, we choose the space $X:=L_{2}(0, \infty)$ with norm $\|\cdot\|_{2}$.

To get differential dependence on initial conditions, we decompose $v$ in (10) into two parts: $u$ which is a solution to a more regular problem and $g$ which is less regular but explicitly known in terms of the Green function $G$ of the operator $A$. Namely, we define $g:[0, \infty) \times[0, \infty) \rightarrow \mathbb{R}$ by

$$
\begin{aligned}
g(x, \eta) & :=A^{-1}(\mu H(\cdot-\eta)(x)) \\
& =\mu \int_{0}^{\infty} G(x, y) H(y-\eta) d y,
\end{aligned}
$$

where $G:[0, \infty) \times[0, \infty) \rightarrow \mathbb{R}$ is a Green's function of $A$ satisfying the Neumann boundary conditions, and $\gamma$ : $[0, \infty) \rightarrow \mathbb{R}$ is given by

$$
\gamma(\eta):=g(\eta, \eta)
$$

If we take a transformation $u(t)(x)=v(x, t)-g(x, \eta(t))$, we have $\left(u_{x}\right)(t)(x)=v_{x}(x, t)-g_{x}(x, \eta(t))$. Since $G_{x}(x, \eta)$ is discontinuous at $x=\eta$, we cannot obtain one step more regular than that of $(10)$.

To overcome this difficulty, let $p(x, t)=v_{x}(x, t)$. Then $p$ satisfies $p_{t}+A p=\mu \delta(x-\eta)$, where $A=-\left(d^{2} / d x^{2}\right)+$ $\mu+1$ with domain $D(A)=\left\{p \in H^{1,2}(\mathbb{R}): p(0, t)=0\right.$, $\left.\lim _{x \rightarrow \infty} p(x, t)=0\right\}$. Define $\widehat{g}:[0, \infty) \times[0, \infty) \rightarrow \mathbb{R}$

$$
\begin{aligned}
\widehat{g}(x, \eta) & :=A^{-1}(\mu \delta(\cdot-\eta)(x)) \\
& =\mu \int_{0}^{\infty} \widehat{G}(x, y) \delta(y-\eta) d y,
\end{aligned}
$$

where $\widehat{G}:[0, \infty) \times[0, \infty) \rightarrow \mathbb{R}$ is a Green's function of $A$ satisfying the Dirichlet boundary conditions, and $\widehat{\gamma}$ : $[0, \infty) \rightarrow \mathbb{R}$ is given by

$$
\widehat{\gamma}(\eta):=\widehat{g}(\eta, \eta) .
$$

We define $j:[0, \infty) \times[0, \infty) \rightarrow \mathbb{R}$,

$$
j(r, \eta):=A_{0}^{-1}(H(\cdot-\eta)(x))=\int_{0}^{\infty} J(x, y) H(y-\eta) d y
$$

and $\alpha:[0, \infty) \times \mathbb{C} \rightarrow \mathbb{C}$

$$
\alpha(\eta):=j(\eta, \eta)
$$

where $J:[0, \infty)^{2} \rightarrow \mathbb{R}$ is a Green's function of $A_{0}$ satisfying the boundary conditions.

Applying the transformations $u(t)(x)=v(x, t)-g(x$, $\eta(t)), z(t)(x)=p(x, t)-\widehat{g}(x, \eta(t))$, and $q(t)(x)=\widehat{w}(x, t)-$ $j(x, \eta(t))$ to $(10)$, we get

$$
\begin{aligned}
u_{t}+A u=\frac{\mu}{\sigma} G(x, \eta) & \\
& \times\left(C\left(u(\eta)+\gamma(\eta) ; a\left(q(\eta)+\alpha(\eta)-s_{0}\right)\right)\right. \\
& \left.+\kappa \chi^{\prime}(u(\eta)+\gamma(\eta))(z(\eta)+\widehat{\gamma}(\eta))\right), \\
z_{t}+A z=- & \frac{1}{\sigma} \frac{\mu}{\eta}(x, \eta) \\
& \times\left(C\left(u(\eta)+\gamma(\eta) ; a\left(q(\eta)+\alpha(\eta)-s_{0}\right)\right)\right. \\
& \left.+\kappa \chi^{\prime}(u(\eta)+\gamma(\eta))(z(\eta)+\widehat{\gamma}(\eta))\right), \\
q_{t}+A_{0} q=\frac{1}{\sigma} J(x, \eta) & \times\left(C\left(u(\eta)+\gamma(\eta) ; a\left(q(\eta)+\alpha(\eta)-s_{0}\right)\right)\right. \\
& \left.+\kappa \chi^{\prime}(u(\eta)+\gamma(\eta))(z(\eta)+\widehat{\gamma}(\eta))\right), \\
& \left.+\kappa \chi^{\prime}(u(\eta)+\gamma(\eta)) \cdot(z(\eta)+\widehat{\gamma}(\eta))\right), \\
\eta^{\prime}(t)=\frac{1}{\sigma}\left(C\left(u(\eta)+\gamma(\eta) ; a\left(q(\eta)+\alpha(\eta)-s_{0}\right)\right)\right. & \\
&
\end{aligned}
$$

Thus, we obtain an abstract evolution equation equivalent to (10):

$$
\begin{gathered}
\frac{d}{d t}(u, z, q, \eta)+\widetilde{A}(u, z, q, \eta)=\frac{1}{\sigma} f(u, z, q, \eta), \\
(u, z, q, \eta)(0)=\left(u_{0}(x), z_{0}(x), q_{0}(x), \eta_{0}\right),
\end{gathered}
$$

where $\widetilde{A}$ is a $4 \times 4$ matrix with the main diagonal entries being the operators $A, A, A_{0}$, and $O$ (the zero operator), and all the other terms are zero. The nonlinear forcing term $f$ is

$$
\begin{array}{r}
f(u, z, q, \eta) \\
=\left(\begin{array}{c}
f_{1}(\eta) \cdot\left(f_{21}(u, z, q, \eta)+f_{22}(u, z, q, \eta)\right) \\
f_{2}(\eta) \cdot\left(f_{21}(u, z, q, \eta)+f_{22}(u, z, q, \eta)\right) \\
f_{3}(\eta) \cdot\left(f_{21}(u, z, q, \eta)+f_{22}(u, z, q, \eta)\right) \\
f_{21}(u, z, q, \eta)+f_{22}(u, z, q, \eta)
\end{array}\right),
\end{array}
$$

where $f_{1}:(0, \infty) \rightarrow X, f_{1}(\eta)(x):=\mu G(x, \eta), f_{2}:$ $(0, \infty) \rightarrow X, f_{2}(\eta)(x):=-(\mu / \eta) \widehat{G}(x, \eta), f_{3}:(0, \infty) \rightarrow$ $X, f_{3}(\eta)(x):=J(x, \eta), f_{21}: W \rightarrow \mathbb{C}, f_{21}(u, z, q, \eta):=$ $C\left(u(\eta)+\gamma(\eta) ; a\left(q(\eta)+\alpha(\eta)-s_{0}\right)\right)$, and $f_{22}: W \rightarrow \mathbb{C}$, 
$f_{22}(u, z, q, \eta):=\kappa \chi^{\prime}(u(\eta)+\gamma(\eta)) \cdot(z(\eta)+\widehat{\gamma}(\eta))$ and $W:=$ $\left\{(u, z, q, \eta) \in C^{1}(0, \infty) \times C^{1}(0, \infty) \times C^{1}(0, \infty) \times(0, \infty):\right.$ $u(\eta)+\gamma(\eta) \in I, z(\eta)+\widehat{\gamma}(\eta) \in I, q(\eta)+\alpha(\eta)-s_{0} \in$ I\} $\subset_{\text {open }} C^{1}(\mathbb{R}) \times C^{1}(\mathbb{R}) \times C^{1}(\mathbb{R}) \times \mathbb{R}$.

The well-posedness of solutions of $(18)$ is shown in $[4,10$, $11]$, using the fractional powers of degree $\theta \in(3 / 4,1]$ of $A$, $A_{0}$, and $\widetilde{A}$ and the methods of the theory of semigroups of operators. Moreover, the nonlinear term $f$ is a continuously differentiable function from $W \cap \widetilde{X}^{\theta}$ to $\widetilde{X}$, where $\widetilde{X}:=D(\widetilde{A})=$ $D(A) \times D(A) \times D\left(A_{0}\right) \times \mathbb{R}, X_{A}^{\theta}:=D\left(A^{\theta}\right), X_{0}^{\theta}:=D\left(A_{0}^{\theta}\right)$, and $\widetilde{X}^{\theta}:=D\left(\widetilde{A}^{\theta}\right)=X_{A}^{\theta} \times X_{A}^{\theta} \times X_{0}^{\theta} \times \mathbb{R}$.

The velocity of $\eta$ is denoted by

$$
\begin{aligned}
C(u(\eta) & \left.+\gamma(\eta) ; a\left(q(\eta)+\alpha(\eta)-s_{0}\right)\right) \\
= & C(S(u, q, \eta)) \\
= & -\frac{1-2 S(u, q, \eta)}{\sqrt{S(u, q, \eta)(1-S(u, q, \eta))}},
\end{aligned}
$$

where $S(u, q, \eta)=u(\eta)+\gamma(\eta)+a\left(q(\eta)+\alpha(\eta)-s_{0}\right)$.

The derivative of $f$ can be obtained following [19].

Lemma 1. The functions $G(\cdot, \eta):(0, \infty) \rightarrow X, \widehat{G}(\cdot, \eta):(0$, $\infty) \rightarrow X, J(\cdot, \eta):(0, \infty) \rightarrow X, C(\cdot): W \rightarrow \mathbb{C}$, and $f: W \rightarrow X \times \mathbb{R}$ are continuously differentiable with derivatives given by

$$
\begin{aligned}
& D f_{21}(u, z, q, \eta)(\widetilde{u}, \widetilde{z}, \widetilde{q}, \tilde{\eta}) \\
& =C_{S}(S(u, q, \eta)) \cdot\left(u^{\prime}(\eta) \tilde{\eta}+\widetilde{u}(\eta)+\gamma^{\prime}(\eta) \tilde{\eta}\right) \\
& +a^{\prime}\left(q(\eta)+\alpha(\eta)-s_{0}\right) \\
& \cdot\left(q^{\prime}(\eta) \hat{\eta}+\widetilde{q}(\eta)+\alpha^{\prime}(\eta) \tilde{\eta}\right), \\
& D f_{22}(u, z, q, \eta)(\widehat{u}, \widehat{z}, \widehat{q}, \widehat{\eta}) \\
& =\kappa \chi^{\prime}(u(\eta)+\gamma(\eta))\left(z^{\prime}(\eta) \hat{\eta}+\widehat{z}(\eta)+\hat{\gamma}^{\prime}(\eta) \hat{\eta}\right), \\
& +\kappa \chi^{\prime \prime}(u(\eta)+\gamma(\eta)) \\
& \left.\cdot\left(\widehat{u}(\eta)+u^{\prime}(\eta) \hat{\eta}+\gamma(\eta) \hat{\eta}\right)\right)(z(\eta)+\widehat{\gamma}(\eta)) \text {, } \\
& \operatorname{Df}(u, z, q, \eta)(\widehat{u}, \widehat{z}, \widehat{q}, \widehat{\eta}) \\
& =\left(f_{21}(u, z, q, \eta)+f_{22}(u, z, q, \eta)\right) \\
& \cdot\left(f_{1}^{\prime}(\eta), f_{2}^{\prime}(\eta), f_{3}^{\prime}(\eta), 0\right) \hat{\eta} \\
& +\left(D f_{21}(u, z, q, \eta)+D f_{22}(u, z, q, \eta)\right)(\widehat{u}, \widehat{z}, \hat{q}, \widehat{\eta}) \\
& \cdot\left(f_{1}(\eta), f_{2}(\eta), f_{3}(\eta), 1\right) .
\end{aligned}
$$

\section{Equilibrium Solutions and Linearization of the Interface Equation}

In this section, we will examine the existence of equilibrium solutions of (18). We look for $\left(u^{*}, z^{*}, q^{*}, \eta^{*}\right) \in D(\widetilde{A}) \cap W$ satisfying the following equations:

$$
\begin{aligned}
& A u= \frac{1}{\sigma} \mu G(x, \eta) \\
& \times\left(C\left(u(\eta)+\gamma(\eta) ; a\left(q(\eta)+\alpha(\eta)-s_{0}\right)\right)\right. \\
&\left.+\kappa \chi^{\prime}(u(\eta)+\gamma(\eta)) \cdot(z(\eta)+\widehat{\gamma}(\eta))\right), \\
& A z=-\frac{1}{\sigma} \frac{\mu}{\eta} \widehat{G}(x, \eta) \\
& \times\left(C\left(u(\eta)+\gamma(\eta) ; a\left(q(\eta)+\alpha(\eta)-s_{0}\right)\right)\right. \\
&\left.+\kappa \chi^{\prime}(u(\eta)+\gamma(\eta)) \cdot(z(\eta)+\widehat{\gamma}(\eta))\right), \\
& A_{0} q= \frac{1}{\sigma} J\left(\cdot, \eta^{*}\right)\left(C\left(u(\eta)+\gamma(\eta) ; a\left(q(\eta)+\alpha(\eta)-s_{0}\right)\right)\right. \\
&\left.+\kappa \chi^{\prime}(u(\eta)+\gamma(\eta)) \cdot(z(\eta)+\widehat{\gamma}(\eta))\right), \\
& 0= C\left(u(\eta)+\gamma(\eta) ; a\left(q(\eta)+\alpha(\eta)-s_{0}\right)\right) \\
&+\kappa \chi^{\prime}(u(\eta)+\gamma(\eta)) \cdot(z(\eta)+\widehat{\gamma}(\eta)), \\
& u^{\prime}(0)= 0=u^{\prime}(\infty), \quad z(0)=0=z(\infty), \\
& q^{\prime}(0)=0=q^{\prime}(\infty) .
\end{aligned}
$$

Theorem 2. Suppose that $(1 / 2)-a\left(1-s_{0}\right)<\mu /(1+\mu)$ and $C^{\prime}\left(\gamma(\eta) ; a\left(\alpha(\eta)-s_{0}\right)\right)+\kappa \chi^{\prime \prime}(\gamma(\eta)) \hat{\gamma}(\eta)-\kappa \sqrt{1+\mu} \chi^{\prime}(\gamma(\eta))>$ 0 for all $\eta>0$. Then (18) has at least one equilibrium solution $\left(0,0,0, \eta^{*}\right)$ for $\kappa<\kappa_{c}$, where $\kappa_{c}$ is a solution of

$$
\begin{aligned}
& C\left(\gamma(\infty) ; a\left(\alpha(\infty)-s_{0}\right)\right) \\
& \quad+\kappa_{c} \chi^{\prime}(\gamma(\infty))\left(\gamma^{\prime}(\infty)+\mu G(\infty, \infty)\right)=0 .
\end{aligned}
$$

The linearization of $f$ at the stationary solution $\left(0,0,0, \eta^{*}\right)$ is

$$
\begin{aligned}
& \operatorname{Df}\left(0,0,0, \eta^{*}\right)(\widehat{u}, \widehat{z}, \widehat{q}, \widehat{\eta}) \\
& =\left(\begin{array}{c}
\mu G\left(\cdot, \eta^{*}\right) Q(\widehat{u}, \widehat{z}, \widehat{q}, \widehat{\eta}) \\
-\frac{\mu}{\eta^{*}} \widehat{G}\left(\cdot, \eta^{*}\right) Q(\widehat{u}, \widehat{z}, \widehat{q}, \widehat{\eta}) \\
j\left(\cdot, \eta^{*}\right) Q(\widehat{u}, \widehat{z}, \widehat{q}, \widehat{\eta}) \\
Q(\widehat{u}, \widehat{z}, \widehat{q}, \widehat{\eta})
\end{array}\right),
\end{aligned}
$$

where $Q(\widehat{u}, \widehat{z}, \widehat{q}, \widehat{\eta})=\left(4+\kappa \chi^{\prime \prime}\left(\gamma\left(\eta^{*}\right) \widehat{\gamma}\left(\eta^{*}\right)\right)\left(\widehat{u}\left(\eta^{*}\right)+\gamma^{\prime}\left(\eta^{*}\right) \widehat{\eta}\right)+\right.$ $\kappa \chi^{\prime}\left(\gamma\left(\eta^{*}\right)\right)\left(\widehat{z}\left(\eta^{*}\right)+\widehat{\gamma}^{\prime}\left(\eta^{*}\right) \widehat{\eta}\right)+4 a^{\prime}\left(\alpha\left(\eta^{*}\right)-s_{0}\right)\left(\widehat{q}\left(\eta^{*}\right)+\alpha^{\prime}\left(\eta^{*}\right) \widehat{\eta}\right)$. The pair $\left(0,0,0, \eta^{*}\right)$ corresponds to a unique steady state $\left(v^{*}, p^{*}, w^{*}, \eta^{*}\right)$ of (10) for $\sigma \neq 0$ with $v^{*}(x)=g\left(x, \eta^{*}\right)$, $p^{*}(x)=\widehat{g}\left(x, \eta^{*}\right)$, and $w^{*}(x)=j\left(x, \eta^{*}\right)-s_{0}$. 
Proof. From the system of (22), we have $u^{*}=0, z^{*}=0$, and $q^{*}=0$. In order to show existence of $\eta^{*}$, we define

$$
\Gamma(\eta, \kappa):=C\left(\gamma(\eta) ; a\left(\alpha(\eta)-s_{0}\right)\right)+\kappa \chi^{\prime}(\gamma(\eta)) \cdot \widehat{\gamma}(\eta) .
$$

Then

$$
\begin{aligned}
\frac{\partial}{\partial \eta} \Gamma(\eta, \kappa)=( & \left.C^{\prime}\left(\gamma(\eta) ; a\left(\alpha(\eta)-s_{0}\right)\right)+\kappa \chi^{\prime \prime}(\gamma(\eta)) \hat{\gamma}(\eta)\right) \\
& \cdot \gamma^{\prime}(\eta)+\kappa \chi^{\prime}(\gamma(\eta)) \widehat{\gamma}^{\prime}(\eta) \\
& +C^{\prime}\left(\gamma(\eta) ; a\left(\alpha(\eta)-s_{0}\right)\right) \\
& \cdot a^{\prime}\left(\alpha(\eta)-s_{0}\right) \cdot \alpha^{\prime}(\eta) .
\end{aligned}
$$

Since $\gamma^{\prime}(\eta)<0$ and $\alpha^{\prime}(\eta)<0$ for all $\eta>0, \Gamma(\eta, \kappa)=0$ is solvable with $\eta^{*}$ if $\Gamma(0, \kappa)>0, \Gamma(\infty, \kappa)<0$, and $(\partial / \partial \eta)$ $\Gamma(\eta, \kappa)<0$, which means that $C\left(\gamma(0) ; a\left(\alpha(0)-s_{0}\right)\right)>0$, $C\left(\gamma(\infty) ; a\left(\alpha(\infty)-s_{0}\right)\right)+\kappa \chi^{\prime}(\gamma(\infty)) \widehat{\gamma}(\infty)<0$, and $C^{\prime}(\gamma(\eta)$; $\left.a\left(\alpha(\eta)-s_{0}\right)\right)+\kappa \chi^{\prime \prime}(\gamma(\eta)) \widehat{\gamma}(\eta)-\kappa \sqrt{1+\mu} \chi^{\prime}(\gamma(\eta))>0$.

Let $\kappa_{c}$ be a solution of

$$
\begin{aligned}
& C\left(\gamma(\infty) ; a\left(\alpha(\infty)-s_{0}\right)\right) \\
& \quad+\kappa_{c} \chi^{\prime}(\gamma(\infty))\left(\gamma^{\prime}(\infty)+\mu G(\infty, \infty)\right)=0 .
\end{aligned}
$$

Then $\Gamma(\infty, \kappa)<\Gamma\left(\infty, \kappa_{c}\right)<\Gamma\left(0, \kappa_{c}\right)$ with $\Gamma\left(\infty, \kappa_{c}\right)=0$. Hence, $\eta^{*}$ exists for $\kappa<\kappa_{c}$.

The formula for $D f\left(0,0,0, \eta^{*}\right)$ follows from the relation $C^{\prime}(1 / 2)=4$, and the corresponding steady state $\left(v^{*}, p^{*}\right.$, $\left.w^{*}, \eta^{*}\right)$ for (10) is obtained by using Theorem 2.1 in [19].

\section{A Hopf Bifurcation}

In this section, we show that there exists a Hopf bifurcation from the curve $\sigma \mapsto\left(0,0,0, \eta^{*}\right)$ of the equilibrium solution. First, let us introduce the following relevant definition.

Definition 3. Under the assumptions of Theorem 2, define (for $1 \geq \theta>3 / 4$ ) the linear operator $B$ from $\widetilde{X}^{\theta}$ to $\widetilde{X}$ by

$$
B:=D f\left(0,0,0, \eta^{*}\right)
$$

We then define $\left(0,0,0, \eta^{*}\right)$ to be a Hopf point for (18) if and only if there exist an $\epsilon_{0}>0$ and a $C^{1}$-curve

$$
\left(-\epsilon_{0}+\tau^{*}, \tau^{*}+\epsilon_{0}\right) \longmapsto(\lambda(\tau), \phi(\tau)) \in \mathbb{C} \times \widetilde{X}_{\mathbf{C}}
$$

$\left(Y_{\mathbf{C}}\right.$ denotes the complexification of the real space $Y$ ) of eigendata for $-\widetilde{A}+\tau B$ with

$$
\text { (i) } \frac{(-\widetilde{A}}{\lambda(\tau)} \frac{+\tau B}{\phi(\tau)},(\phi(\tau))=\lambda(\tau) \phi(\tau),(-\widetilde{A}+\tau B)(\overline{\phi(\tau)})=
$$

(ii) $\lambda\left(\tau^{*}\right)=i \beta$ with $\beta>0$, (iii) $\operatorname{Re}(\lambda) \neq 0$ for all $\lambda$ in the spectrum of $\left(-\widetilde{A}+\tau^{*} B\right) \backslash$ $\{ \pm i \beta\}$,

(iv) $\operatorname{Re} \lambda^{\prime}\left(\tau^{*}\right) \neq 0$ (transversality),

where $\tau=1 / \sigma$.

Next, we check (18) for the Hopf points. For this, we solve the eigenvalue problem:

$$
-\widetilde{A}(u, z, q, \eta)+\tau B(u, z, q, \eta)=\lambda I_{4}(u, z, q, \eta),
$$

where $I_{4}$ is a $4 \times 4$ identity matrix. This is equivalent to

$$
\begin{aligned}
(A+\lambda) u= & \tau \mu G\left(\cdot, \eta^{*}\right) \\
\times & \left(d_{2}\left(u\left(\eta^{*}\right)+\gamma^{\prime}\left(\eta^{*}\right) \eta\right)\right. \\
& +\kappa d_{1}\left(z\left(\eta^{*}\right)+\widehat{\gamma}^{\prime}\left(\eta^{*}\right) \eta\right) \\
& \left.+a_{1}\left(q\left(\eta^{*}\right)+\alpha^{\prime}\left(\eta^{*}\right)\right)\right), \\
(A+\lambda) z=- & \frac{\tau \mu}{\eta^{*}} \widehat{G}\left(\cdot, \eta^{*}\right) \\
\times & \left(d_{2}\left(u\left(\eta^{*}\right)+\gamma^{\prime}\left(\eta^{*}\right) \eta\right)\right. \\
& +\kappa d_{1}\left(z\left(\eta^{*}\right)+\widehat{\gamma}^{\prime}\left(\eta^{*}\right) \eta\right) \\
& \left.+a_{1}\left(q\left(\eta^{*}\right)+\alpha^{\prime}\left(\eta^{*}\right)\right)\right), \\
\left(A_{0}+\lambda\right) q= & \left(\cdot, \eta^{*}\right) \\
\times & \left(d_{2}\left(u\left(\eta^{*}\right)+\gamma^{\prime}\left(\eta^{*}\right) \eta\right)\right. \\
& +\kappa d_{1}\left(z\left(\eta^{*}\right)+\widehat{\gamma}^{\prime}\left(\eta^{*}\right) \eta\right) \\
& \left.+a_{1}\left(q\left(\eta^{*}\right)+\alpha^{\prime}\left(\eta^{*}\right)\right)\right), \\
& \left.+a_{1}\left(q\left(\eta^{*}\right)+\alpha^{\prime}\left(\eta^{*}\right)\right)\right), \\
& +\kappa d_{1}\left(z\left(\eta^{*}\right)+\gamma^{\prime}\left(\eta^{*}\right) \eta\right) \\
& \\
& \\
&
\end{aligned}
$$

where $d_{1}=\chi^{\prime}\left(\gamma\left(\eta^{*}\right)\right), d_{2}=4+\kappa \chi^{\prime \prime}\left(\gamma\left(\eta^{*}\right)\right) \widehat{\gamma}\left(\eta^{*}\right)$, and $a_{1}=$ $4 a^{\prime}\left(\alpha\left(\eta^{*}\right)-s_{0}\right)$.

In the following theorem, we show that an equilibrium solution is a Hopf point.

Theorem 4. Suppose that $(1 / 2)-a\left(1-s_{0}\right)<(\mu /(1+\mu))$ and $C^{\prime}\left(\gamma(\eta) ; a\left(\alpha(\eta)-s_{0}\right)\right)+\kappa \chi^{\prime \prime}(\gamma(\eta)) \widehat{\gamma}(\eta)>\kappa \sqrt{1+\mu} \chi^{\prime}(\gamma(\eta))$ for all $\eta>0$. Assume that $C^{\prime}\left(\gamma\left(\eta^{*}\right) ; a\left(\alpha\left(\eta^{*}\right)-s_{0}\right)\right)+$ $\kappa \chi^{\prime \prime}\left(\gamma\left(\eta^{*}\right)\right) \hat{\gamma}\left(\eta^{*}\right)>\left(\kappa / \eta^{*}\right) \chi^{\prime}\left(\gamma\left(\eta^{*}\right)\right)$. Additionally, suppose that the operator $-\widetilde{A}+\tau^{*} B$ has a unique pair $\{ \pm i \beta\}, \beta>$ 0 of purely imaginary eigenvalues for some $\tau^{*}>0$. Then, $\left(0,0,0, \eta^{*}, \tau^{*}\right)$ is a Hopf point for (18).

Proof. We assume, without loss of generality, that $\beta>0$, and $\Phi^{*}$ is the (normalized) eigenfunction of $-\widetilde{A}+\tau^{*} B$ with 
eigenvalue $i \beta$. We have to show that $\left(\Phi^{*}, i \beta\right)$ can be extended to a $C^{1}$-curve $\tau \mapsto(\Phi(\tau), \lambda(\tau))$ of eigendata for $-\widetilde{A}+\tau B$ with $\operatorname{Re}\left(\lambda^{\prime}\left(\tau^{*}\right)\right) \neq 0$.

For this, let $\Phi^{*}=\left(\psi_{0}, z_{0}, q_{0}, \eta_{0}\right) \in D(A) \times D(A) \times$ $D\left(A_{0}\right) \times \mathbb{R}$. First, we note that $\eta_{0} \neq 0$. Otherwise, by (31),
$(A+i \beta) \psi_{0}=\mu i \beta \eta_{0} G\left(\cdot, \eta^{*}\right)=0$ and $(A+i \beta) z_{0}=$ $-\left(\mu / \eta^{*}\right) i \beta \eta_{0} \widehat{G}\left(\cdot, \eta^{*}\right)=0$, which is not possible because $A$ is symmetric. So, without loss of generality, let $\eta_{0}=1$. Then $E\left(\psi_{0}, z_{0}, q_{0}, i \beta, \tau^{*}\right)=0$ by (31), where

$$
E(u, z, q, \lambda, \tau):=\left(\begin{array}{c}
E: D(A)_{\mathbf{C}} \times D(A)_{\mathbf{C}} \times D\left(A_{0}\right)_{\mathbf{C}} \times \mathbb{C} \times \mathbb{R} \longrightarrow X_{\mathbf{C}} \times X_{\mathbf{C}} \times X_{\mathbf{C}} \times \mathbb{C}, \\
(A+\lambda) u-\tau \mu G\left(\cdot, \eta^{*}\right)\left(d_{2}\left(u\left(\eta^{*}\right)+\gamma^{\prime}\left(\eta^{*}\right)\right)+\kappa d_{1}\left(z\left(\eta^{*}\right)+\widehat{\gamma}^{\prime}\left(\eta^{*}\right)+a_{1} \cdot\left(q\left(\eta^{*}\right)+\alpha\left(\eta^{*}\right)\right)\right)\right. \\
\left(A_{0}+\lambda\right) q-\tau \frac{\mu}{\eta^{*}} \widehat{G}\left(\cdot, \eta^{*}\right)\left(d_{2}\left(u\left(\eta^{*}\right)+\gamma^{\prime}\left(\eta^{*}\right)\right)+\kappa d_{1}\left(z\left(\eta^{*}\right)+\widehat{\gamma}^{\prime}\left(\eta^{*}\right)+a_{1} \cdot\left(q\left(\eta^{*}\right)+\gamma^{\prime}\left(\eta^{*}\right)\right)+\kappa d_{1}\left(z\left(\eta^{*}\right)+\widehat{\gamma}^{\prime}\left(\eta^{*}\right)\right)+a_{1} \cdot\left(q\left(\eta^{*}\right)+\alpha\left(\eta^{*}\right)\right)\right)\right. \\
\lambda-\tau\left(d_{2}\left(u\left(\eta^{*}\right)+\gamma^{\prime}\left(\eta^{*}\right)\right)+\kappa d_{1}\left(z\left(\eta^{*}\right)+\widehat{\gamma}^{\prime}\left(\eta^{*}\right)+a_{1} \cdot\left(q\left(\eta^{*}\right)+\alpha\left(\eta^{*}\right)\right)\right)\right.
\end{array}\right) .
$$

The equation $E(u, z, q, \lambda, \tau)=0$ is equivalent to $\lambda$ being an eigenvalue of $-\widetilde{A}+\tau B$ with eigenfunction $(u, z, q, 1)$. We will apply the implicit function theorem to $E$. For this, we check that $E$ is of $C^{1}$-class and that

$$
\begin{aligned}
& D_{(u, z, q, \lambda)} E\left(\psi_{0}, z_{0}, q_{0}, i \beta, \tau^{*}\right) \\
& \in L\left(D(A)_{\mathbf{C}} \times D(A)_{\mathbf{C}} \times D\left(A_{0}\right)_{\mathbf{C}} \times \mathbb{C} \times \mathbb{R},\right. \\
& \left.\quad X_{\mathbf{C}} \times X_{\mathbf{C}} \times X_{\mathbf{C}} \times \mathbb{C}\right)
\end{aligned}
$$

is an isomorphism. In addition, the mapping

$$
D_{(u, z, q, \lambda)} E\left(\psi_{0}, z_{0}, q_{0}, i \beta, \tau^{*}\right)(\widehat{u}, \widehat{z}, \widehat{q}, \widehat{\lambda})=\left(\begin{array}{c}
(A+i \beta) \hat{u}+\widehat{\lambda} \psi_{0}-\tau^{*} \mu G\left(\cdot \eta^{*}\right)\left(d_{2} \widehat{u}\left(\eta^{*}\right)+\kappa d_{1} \widehat{z}\left(\eta^{*}\right)+a_{1} \widehat{q}\left(\eta^{*}\right)\right) \\
(A+i \beta) \widehat{z}+\hat{\lambda} z_{0}+\tau^{*} \frac{\mu}{\eta^{*}} \widehat{G}\left(\cdot, \eta^{*}\right)\left(d_{2} \widehat{u}\left(\eta^{*}\right)+\kappa d_{1} \widehat{z}\left(\eta^{*}\right)+a_{1} \widehat{q}\left(\eta^{*}\right)\right) \\
\left(A_{0}+i \beta\right) \hat{q}+\widehat{\lambda} q_{0}-\tau^{*} J\left(\cdot, \eta^{*}\right)\left(d_{2} \widehat{u}\left(\eta^{*}\right)+\kappa d_{1} \widehat{z}\left(\eta^{*}\right)+a_{1} \widehat{q}\left(\eta^{*}\right)\right) \\
\widehat{\lambda}-\tau^{*}\left(d_{2} \widehat{u}\left(\eta^{*}\right)+\kappa d_{1} \widehat{z}\left(\eta^{*}\right)+a_{1} \widehat{q}\left(\eta^{*}\right)\right)
\end{array}\right)
$$

is a compact perturbation of the mapping

$$
(\widehat{u}, \widehat{z}, \widehat{q}, \widehat{\lambda}) \longmapsto\left((A+i \beta) \widehat{u},(A+i \beta) \widehat{z},\left(A_{0}+i \beta\right) \hat{q}, \widehat{\lambda}\right)
$$

which is invertible. Thus, $D_{(u, z, q, \lambda)} E\left(\psi_{0}, z_{0}, q_{0}, i \beta, \tau^{*}\right)$ is a Fredholm operator of index 0 . Therefore, in order to verify (33), it suffices to show that the system of equations

$$
D_{(u, z, q, \lambda)} E\left(\psi_{0}, z_{0}, q_{0}, i \beta, \tau^{*}\right)(\widehat{u}, \widehat{z}, \widehat{q}, \widehat{\lambda})=0
$$

which is equivalent to

$$
\begin{aligned}
(A+i \beta) \widehat{u}+\widehat{\lambda} \psi_{0}= & \tau^{*} \mu G\left(\cdot, \eta^{*}\right) \\
& \times\left(d_{2} \widehat{u}\left(\eta^{*}\right)+\kappa d_{1} \widehat{z}\left(\eta^{*}\right)+a_{1} \widehat{q}\left(\eta^{*}\right)\right), \\
(A+i \beta) \widehat{z}+\widehat{\lambda} \xi_{0}= & -\tau^{*} \frac{\mu}{\eta^{*}} \widehat{G}\left(\cdot, \eta^{*}\right) \\
& \times\left(d_{2} \widehat{u}\left(\eta^{*}\right)+\kappa d_{1} \widehat{z}\left(\eta^{*}\right)+a_{1} \widehat{q}\left(\eta^{*}\right)\right),
\end{aligned}
$$

$$
\begin{aligned}
&\left(A_{0}+i \beta\right) \widehat{q}+\widehat{\lambda} q_{0}= \tau^{*} J\left(\cdot, \eta^{*}\right) \\
& \times\left(d_{2} \widehat{u}\left(\eta^{*}\right)+\kappa d_{1} \widehat{z}\left(\eta^{*}\right)+a_{1} \widehat{q}\left(\eta^{*}\right)\right), \\
& \widehat{\lambda}=\tau^{*}\left(d_{2} \widehat{u}\left(\eta^{*}\right)+\kappa d_{1} \widehat{z}\left(\eta^{*}\right)+a_{1} \widehat{q}\left(\eta^{*}\right)\right)
\end{aligned}
$$

necessarily implies that $\widehat{u}=0, \widehat{z}=0, \widehat{q}=0$, and $\widehat{\lambda}=0$. If we define $\phi:=\psi_{0}-\mu G\left(\cdot, \eta^{*}\right), \xi:=z_{0}+\left(\mu / \eta^{*}\right) \widehat{G}\left(\cdot, \eta^{*}\right)$, and $\rho=q_{0}-J\left(\cdot, \eta^{*}\right)$, then (37) becomes

$$
(A+i \beta) \widehat{u}+\widehat{\lambda} \phi=0,
$$

$$
(A+i \beta) \widehat{z}+\hat{\lambda} \xi=0
$$

$$
\left(A_{0}+i \beta\right) \hat{q}+\hat{\lambda} \rho=0
$$

$$
\frac{\widehat{\lambda}}{\tau^{*}}=d_{2} \widehat{u}\left(\eta^{*}\right)+\kappa d_{1} \widehat{z}\left(\eta^{*}\right)+a_{1} \widehat{q}\left(\eta^{*}\right) .
$$


On the other hand, since $E\left(\psi_{0}, z_{0}, q_{0}, i \beta, \tau^{*}\right)=0, \phi, \xi$ and $\rho$ are solutions to the equations, we have

$$
\begin{gathered}
(A+i \beta) \phi=-\mu \delta_{\eta^{*}}, \\
(A+i \beta) \xi=\frac{\mu}{\eta^{*}} \delta_{\eta^{*}}, \\
\left(A_{0}+i \beta\right) \rho=-\delta_{\eta^{*}}, \\
\frac{i \beta}{\tau^{*}}=d_{2}\left(\phi\left(\eta^{*}\right)+\mu G\left(\eta^{*}, \eta^{*}\right)+\gamma^{\prime}\left(\eta^{*}\right)\right) \\
+\kappa d_{1}\left(\xi\left(\eta^{*}\right)-\frac{\mu}{\eta^{*}} \widehat{G}\left(\eta^{*}, \eta^{*}\right)+\widehat{\gamma}^{\prime}\left(\eta^{*}\right)\right) \\
+a_{1}\left(\rho\left(\eta^{*}\right)+J\left(\eta^{*}, \eta^{*}\right)+\alpha^{\prime}\left(\eta^{*}\right)\right) .
\end{gathered}
$$

Multiplying (39) and (43) by $\phi$ and (38) and (42) by $\xi$ and subtracting one from the other, we obtain

$$
\widehat{u}\left(\eta^{*}\right)=-\eta^{*} \widehat{z}\left(\eta^{*}\right), \quad \phi\left(\eta^{*}\right)=-\eta^{*} \xi\left(\eta^{*}\right) .
$$

Multiplying (38) by $d_{2} \bar{\phi},(39)$ by $-\eta^{*} \kappa d_{1} \bar{\rho}$, and (40) by $a_{1} \bar{\rho}$ and adding the resultants to each, we have

$$
\begin{aligned}
& -d_{2} \mu \widehat{u}\left(\eta^{*}\right)-\kappa d_{1} \mu \widehat{z}\left(\eta^{*}\right)-a_{1} \widehat{q}\left(\eta^{*}\right) \\
& +\widehat{\lambda}\left(d_{2}\|\phi\|^{2}-\eta^{*} \kappa d_{1}\|\xi\|^{2}+a_{1}\|\rho\|^{2}\right) \\
& \quad+2 i \beta \int\left(d_{2} \widehat{u} \bar{\phi}-\eta^{*} \kappa d_{1} \widehat{z} \bar{\xi}+a_{1} \widehat{q} \bar{\rho}\right)=0 .
\end{aligned}
$$

Multiplying (42) by $d_{2} \bar{\phi}$, (43) by $-\eta^{*} \kappa d_{1} \bar{\rho}$, and (44) by $a_{1} \bar{\rho}$ and adding the resultants to each, we obtain

$$
\begin{aligned}
d_{2}\left\|A^{1 / 2} \phi\right\|^{2}-\eta^{*} \kappa d_{1}\left\|A^{1 / 2} \xi\right\|^{2}+a_{1}\left\|A_{0}^{1 / 2} \rho\right\|^{2} \\
+i \beta\left(d_{2}\|\phi\|^{2}-\eta^{*} \kappa d_{1}\|\xi\|^{2}+a_{1}\|\rho\|^{2}\right) \\
=-d_{2} \mu \overline{\phi\left(\eta^{*}\right)}-\mu \kappa d_{1} \overline{\xi\left(\eta^{*}\right)}-a_{1} \overline{\rho\left(\eta^{*}\right)} .
\end{aligned}
$$

From (45), we get

$$
\frac{\mu}{\tau^{*}}=d_{2}\|\phi\|^{2}-\eta^{*} \kappa d_{1}\|\xi\|^{2}+a_{1}\|\rho\|^{2}
$$

and thus (47) implies that

$$
\int\left(d_{2} \widehat{u} \bar{\phi}-\eta^{*} \kappa d_{1} \widehat{z} \bar{\xi}+a_{1} \widehat{q} \bar{\rho}\right)=0
$$

Now, multiplying (38) by $d_{2} \overline{\widehat{u}},(42)$ by $-\eta^{*} \kappa d_{1} \overline{\widehat{z}}$, and (40) by $a_{1} \overline{\hat{q}}$ and adding the resultants to each, we have

$$
\begin{gathered}
\left(d_{2}\left\|A^{1 / 2} \widehat{u}\right\|^{2}-\eta^{*} \kappa d_{1}\left\|A^{1 / 2} \widehat{z}\right\|^{2}+a_{1}\left\|A_{0}^{1 / 2} \hat{q}\right\|^{2}\right) \\
+i \beta\left(d_{2}\|\widehat{u}\|^{2}-\eta^{*} \kappa d_{1}\|\widehat{z}\|^{2}+a_{1}\|\hat{q}\|^{2}\right) \\
+\hat{\lambda} \int\left(d_{2} \phi \overline{\widehat{u}}-\eta^{*} \kappa d_{1} \xi \overline{\widehat{z}}+a_{1} \rho \overline{\widehat{q}}\right)=0 .
\end{gathered}
$$

From (50), we get

$$
\begin{gathered}
d_{2}\left\|A^{1 / 2} \widehat{u}\right\|^{2}-\eta^{*} \kappa d_{1}\left\|A^{1 / 2} \hat{z}\right\|^{2}+a_{1}\left\|A_{0}^{1 / 2} \hat{q}\right\|^{2}=0 \\
d_{2}\|\widehat{u}\|^{2}-\eta^{*} \kappa d_{1}\|\widehat{z}\|^{2}+a_{1}\|\widehat{q}\|^{2}=0 .
\end{gathered}
$$

Multiplying (42) by $\bar{\phi}$ and (43) by $\bar{\xi}$, we get

$$
\begin{aligned}
& \left\|A^{1 / 2} \phi\right\|^{2}+i \beta\|\phi\|^{2}=-\mu \bar{\phi}\left(\eta^{*}\right), \\
& \left\|A^{1 / 2} \xi\right\|^{2}+i \beta\|\xi\|^{2}=\frac{\mu}{\eta^{*}} \bar{\xi}\left(\eta^{*}\right),
\end{aligned}
$$

and applying (46) to the above equation, we have

$$
\left\|A^{1 / 2} \phi\right\|^{2}=\left(\eta^{*}\right)^{2}\left\|A^{1 / 2} \xi\right\|^{2}, \quad\|\phi\|^{2}=\left(\eta^{*}\right)^{2}\|\xi\|^{2} .
$$

Now, multiplying (38) by $2 i \beta \overline{\widehat{u}}$ and (42) by $\hat{\lambda} \overline{\widehat{u}}$ and subtracting the resultants to each other, we obtain

$$
\begin{aligned}
2 i \beta( & \left.\left\|A^{1 / 2} \widehat{u}\right\|^{2}-\left(\eta^{*}\right)^{2}\left\|A^{1 / 2} \widehat{z}\right\|^{2}\right) \\
& -2 \beta^{2}\left(\|\widehat{u}\|^{2}-\left(\eta^{*}\right)^{2}\|\widehat{z}\|^{2}\right)+\hat{\lambda}\left(\|\phi\|^{2}-\left(\eta^{*}\right)^{2}\|\xi\|^{2}\right) .
\end{aligned}
$$

Applying (54) to the above equation, we have

$$
\left\|A^{1 / 2} \widehat{u}\right\|^{2}-\left(\eta^{*}\right)^{2}\left\|A^{1 / 2} \widehat{z}\right\|^{2}=0, \quad\|\widehat{u}\|^{2}-\left(\eta^{*}\right)^{2}\|\widehat{z}\|^{2}=0,
$$

and thus (52) implies that

$$
\left(d_{2}-\frac{\kappa d_{1}}{\eta^{*}}\right)\|\widehat{u}\|^{2}+a_{1}\|\widehat{q}\|^{2}=0
$$

Since $d_{2}-\left(\kappa d_{1} / \eta^{*}\right)>0$ and $a_{1}>0$, we have $\widehat{u}=0$ and $\widehat{q}=0$, and so, $\widehat{z}=0$ and $\widehat{\lambda}=0$.

Theorem 5. Under the same condition as in Theorem 4, $(0,0$, $\left.0, \eta^{*}, \tau^{*}\right)$ satisfies the transversality condition. Hence, this is a Hopf point for (18).

Proof. By the implicit differentiation of $E\left(\psi_{0}(\tau), z_{0}(\tau)\right.$, $\left.q_{0}(\tau), \lambda(\tau), \tau\right)=0$, we find 


$$
\begin{aligned}
D_{(u, z, q, \lambda)} E\left(\psi_{0}, z_{0}, q_{0}, i \beta, \tau^{*}\right)\left(\psi_{0}^{\prime}\left(\tau^{*}\right), z_{0}^{\prime}\left(\tau^{*}\right), q_{0}^{\prime}\left(\tau^{*}\right), \lambda^{\prime}\left(\tau^{*}\right)\right) \\
=\left(\begin{array}{c}
\mu G\left(\cdot, \eta^{*}\right)\left(d_{2}\left(\psi_{0}\left(\eta^{*}\right)+\gamma^{\prime}\left(\eta^{*}\right)\right)+\kappa d_{1}\left(z_{0}\left(\eta^{*}\right)+\widehat{\gamma}^{\prime}\left(\eta^{*}\right)\right)+a_{1}\left(q_{0}\left(\eta^{*}\right)+\alpha\left(\eta^{*}\right)\right)\right. \\
-\frac{\mu}{\eta^{*}}\left(\cdot, \eta^{*}\right)\left(d_{2}\left(\psi_{0}\left(\eta^{*}\right)+\gamma^{\prime}\left(\eta^{*}\right)\right)+\kappa d_{1}\left(z_{0}\left(\eta^{*}\right)+\widehat{\gamma}^{\prime}\left(\eta^{*}\right)\right)+a_{1}\left(q_{0}\left(\eta^{*}\right)+\alpha\left(\eta^{*}\right)\right)\right. \\
J\left(\cdot, \eta^{*}\right)\left(d_{2}\left(\psi_{0}\left(\eta^{*}\right)+\gamma^{\prime}\left(\eta^{*}\right)\right)+\kappa d_{1}\left(z_{0}\left(\eta^{*}\right)+\hat{\gamma}^{\prime}\left(\eta^{*}\right)\right)+a_{1}\left(q_{0}\left(\eta^{*}\right)+\alpha\left(\eta^{*}\right)\right)\right. \\
d_{2}\left(\psi_{0}\left(\eta^{*}\right)+\gamma^{\prime}\left(\eta^{*}\right)\right)+\kappa d_{1}\left(z_{0}\left(\eta^{*}\right)+\widehat{\gamma}^{\prime}\left(\eta^{*}\right)\right)+a_{1}\left(q_{0}\left(\eta^{*}\right)+\alpha\left(\eta^{*}\right)\right)
\end{array}\right) .
\end{aligned}
$$

This means that the functions $\widetilde{u}:=\psi_{0}^{\prime}\left(\tau^{*}\right), \widetilde{z}:=z_{0}^{\prime}\left(\tau^{*}\right), \widetilde{q}:=$ $q_{0}^{\prime}\left(\tau^{*}\right)$, and $\tilde{\lambda}:=\lambda^{\prime}\left(\tau^{*}\right)$ satisfy the equations

$$
\begin{aligned}
&(A+i \beta) \tilde{u}+\widetilde{\lambda} \psi_{0}-\tau^{*} \mu G\left(\cdot, \eta^{*}\right) \\
& \times\left(d_{2} \widetilde{u}\left(\eta^{*}\right)+\kappa d_{1} \widetilde{z}\left(\eta^{*}\right)+a_{1} \widetilde{q}\left(\eta^{*}\right)\right) \\
&= \mu G\left(\cdot, \eta^{*}\right)\left(d_{2}\left(\psi_{0}\left(\eta^{*}\right)+\gamma^{\prime}\left(\eta^{*}\right)\right)\right. \\
&+\kappa d_{1}\left(z_{0}\left(\eta^{*}\right)+\widehat{\gamma}^{\prime}\left(\eta^{*}\right)\right)+a_{1}\left(q_{0}\left(\eta^{*}\right)+\alpha\left(\eta^{*}\right)\right), \\
&(A+i \beta) \widetilde{z}+\widetilde{\lambda} \xi_{0}+\tau^{*} \frac{\mu}{\eta^{*}} \widehat{G}\left(\cdot, \eta^{*}\right) \\
& \times\left(d_{2} \widetilde{u}\left(\eta^{*}\right)+\kappa d_{1} \widetilde{z}\left(\eta^{*}\right)+a_{1} \widetilde{q}\left(\eta^{*}\right)\right) \\
&=-\frac{\mu}{\eta^{*}} \widehat{G}\left(\cdot, \eta^{*}\right)\left(d_{2}\left(\psi_{0}\left(\eta^{*}\right)+\gamma^{\prime}\left(\eta^{*}\right)\right)\right. \\
&+\kappa d_{1}\left(z_{0}\left(\eta^{*}\right)+\widehat{\gamma}^{\prime}\left(\eta^{*}\right)\right)+a_{1}\left(q_{0}\left(\eta^{*}\right)+\alpha\left(\eta^{*}\right)\right), \\
&\left(A_{0}+\right.i \beta) \widetilde{q}+\widetilde{\lambda} \rho_{0}-\tau^{*} J\left(\cdot, \eta^{*}\right) \\
& \times\left(d_{2} \widetilde{u}\left(\eta^{*}\right)+\kappa d_{1} \widetilde{z}\left(\eta^{*}\right)+a_{1} \widetilde{q}\left(\eta^{*}\right)\right) \\
&= J\left(\cdot, \eta^{*}\right)\left(d_{2}\left(\psi_{0}\left(\eta^{*}\right)+\gamma^{\prime}\left(\eta^{*}\right)\right)\right. \\
&+\kappa d_{1}\left(z_{0}\left(\eta^{*}\right)+\widehat{\gamma}^{\prime}\left(\eta^{*}\right)\right)+a_{1}\left(q_{0}\left(\eta^{*}\right)+\alpha\left(\eta^{*}\right)\right), \\
& \tilde{\lambda}-\tau^{*}\left(d_{2} \tilde{u}\left(\eta^{*}\right)+\kappa d_{1} z\left(\eta^{*}\right)+a_{1} \widetilde{q}\left(\eta^{*}\right)\right) \\
&=d_{2}\left(\psi_{0}\left(\eta^{*}\right)+\gamma^{\prime}\left(\eta^{*}\right)\right) \\
&+\kappa d_{1}\left(z_{0}\left(\eta^{*}\right)+\widehat{\gamma}^{\prime}\left(\eta^{*}\right)\right)+a_{1}\left(q_{0}\left(\eta^{*}\right)+\alpha\left(\eta^{*}\right)\right) .
\end{aligned}
$$

By letting $\phi:=\psi_{0}-\mu G\left(\cdot, \eta^{*}\right), \quad \xi=z_{0}+\left(\mu / \eta^{*}\right) \widehat{G}\left(\cdot, \eta^{*}\right)$, and $\rho=q_{0}-J\left(\cdot, \eta^{*}\right)$ as before, we obtain

$$
\begin{gathered}
(A+i \beta) \tilde{u}+\tilde{\lambda} \phi=0, \\
(A+i \beta) \tilde{z}+\tilde{\lambda} \xi=0, \\
\left(A_{0}+i \beta\right) \tilde{q}+\tilde{\lambda} \rho=0, \\
\tilde{\lambda}-\tau^{*}\left(d_{2} \widetilde{u}\left(\eta^{*}\right)+\kappa d_{1} \tilde{z}\left(\eta^{*}\right)+a_{1} \widetilde{q}\left(\eta^{*}\right)\right)=\frac{i \beta}{\tau^{*}} .
\end{gathered}
$$

Multiplying (60) by $d_{2} \bar{\phi}$, (61) by $-\eta^{*} \kappa d_{1} \bar{\xi}$, and (62) by $a_{1} \bar{\rho}$ and adding the resultants to each, we obtain

$$
\begin{aligned}
& -d_{2} \mu \tilde{u}\left(\eta^{*}\right)-\kappa d_{1} \mu \widetilde{z}\left(\eta^{*}\right)-a_{1} \widetilde{q}\left(\eta^{*}\right) \\
& +\widetilde{\lambda}\left(d_{2}\|\phi\|^{2}-\eta^{*} \kappa d_{1}\|\xi\|^{2}+a_{1}\|\rho\|^{2}\right) \\
& +2 i \beta \int\left(d_{2} \widetilde{u} \bar{\phi}-\eta^{*} \kappa d_{1} \bar{z} \bar{\xi}+a_{1} \widetilde{q} \bar{\rho}\right)=0 .
\end{aligned}
$$

From (49) and (63), the above equation implies that

$$
i \beta \frac{\mu}{\left(\tau^{*}\right)^{2}}+2 i \beta \int\left(d_{2} \widetilde{u} \bar{\phi}-\eta^{*} \kappa d_{1} \widetilde{z} \bar{\xi}+a_{1} \widetilde{q} \bar{\rho}\right)=0 .
$$

Multiplying (60) by $d_{2} \overline{\tilde{u}}$, (61) by $-\eta^{*} \kappa d_{1} \overline{\tilde{z}}$ and (62) by $a_{1} \overline{\tilde{q}}$ and adding the resultants to each, we have

$$
\begin{aligned}
& d_{2}\left\|A^{1 / 2} \widetilde{u}\right\|^{2}-\eta^{*} \kappa d_{1}\left\|A^{1 / 2} \tilde{z}\right\|^{2} \\
& \quad+a_{1}\left\|A_{0}^{1 / 2} \widetilde{q}\right\|^{2} \\
& +i \beta\left(d_{2}\|\tilde{u}\|^{2}-\eta^{*} \kappa d_{1}\|\tilde{z}\|^{2}+a_{1}\|\widetilde{q}\|^{2}\right) \\
& \quad+\tilde{\lambda} \int\left(d_{2} \tilde{u} \bar{\phi}-\eta^{*} \kappa d_{1} \bar{z} \bar{\xi}+a_{1} \widetilde{q} \bar{\rho}\right)=0 .
\end{aligned}
$$

From (65), we have

$$
\begin{aligned}
& d_{2}\left\|A^{1 / 2} \widetilde{u}\right\|^{2}-\eta^{*} \kappa d_{1}\left\|A^{1 / 2} \widetilde{z}\right\|^{2}+a_{1}\left\|A_{0}^{1 / 2} \widetilde{q}\right\|^{2} \\
& \quad+i \beta\left(d_{2}\|\widetilde{u}\|^{2}-\eta^{*} \kappa d_{1}\|\tilde{z}\|^{2}+a_{1}\|\widetilde{q}\|^{2}\right)=\tilde{\lambda} \frac{\mu}{2\left(\tau^{*}\right)^{2}},
\end{aligned}
$$

and the real part is

$$
d_{2}\left\|A^{1 / 2} \widetilde{u}\right\|^{2}-\eta^{*} \kappa d_{1}\left\|A^{1 / 2} \widetilde{z}\right\|^{2}+a_{1}\left\|A_{0}^{1 / 2} \tilde{q}\right\|^{2}=\frac{\mu}{2\left(\tau^{*}\right)^{2}} \operatorname{Re} \tilde{\lambda} .
$$

Now, multiplying (60) by $2 i \beta \overline{\tilde{u}}$ and (61) by $\tilde{\lambda} \overline{\tilde{u}}$ and applying (54) to resultants, we obtain

$$
\left\|A^{1 / 2} \widetilde{u}\right\|^{2}-\left(\eta^{*}\right)^{2}\left\|A^{1 / 2} \widetilde{z}\right\|^{2}=0, \quad\|\widetilde{u}\|^{2}-\left(\eta^{*}\right)^{2}\|\widetilde{z}\|^{2}=0,
$$

and thus (68) implies that

$$
\frac{\mu}{2\left(\tau^{*}\right)^{2}} \operatorname{Re} \tilde{\lambda}=\left(d_{2}-\frac{\kappa d_{1}}{\eta^{*}}\right)\left\|A^{1 / 2} \widetilde{u}\right\|^{2}+a_{1}\left\|A_{0}^{1 / 2} \tilde{q}\right\|^{2}
$$


which is positive since $d_{2}-\left(\kappa d_{1} / \eta^{*}\right)>0$ and $a_{1}>0$. We have $\operatorname{Re} \lambda^{\prime}\left(\tau^{*}\right)>0$ for $\beta>0$, and thus, by the Hopfbifurcation theorem in [19], there exists a family of periodic solutions which bifurcates from the stationary solution as $\tau$ passes $\tau^{*}$.

Now, we show that there exists a unique $\tau^{*}>0$ such that $\left(0,0, \eta^{*}, \tau^{*}\right)$ is a Hopf point; thus $\tau^{*}$ is the origin of a branch of nontrivial periodic orbits.

Lemma 6. Suppose that $d_{2}-\left(\kappa d_{1} / \eta^{*}\right)>0$. Let $G_{\beta}, \widehat{G}_{\beta}$, and $J_{\beta}$ be Green functions of the differential operators $A+i \beta$, $A+i / \beta$ and $A_{0}+i \beta$ satisfying (42), (43), and (44), respectively. Then, $d_{2} \operatorname{Re}\left(G_{\beta}\left(\eta^{*}, \eta^{*}\right)\right)-\left(\kappa d_{1} / \eta^{*}\right) \operatorname{Re}\left(\widehat{G}_{\beta}\left(\eta^{*}, \eta^{*}\right)\right)$ and $\operatorname{Re}\left(J_{\beta}\left(\eta^{*}, \eta^{*}\right)\right)$ are strictly decreasing in $\beta \in \mathbb{R}^{+}$with

$$
\operatorname{Re} G_{0}\left(\eta^{*}, \eta^{*}\right)=G\left(\eta^{*}, \eta^{*}\right), \quad \lim _{\beta \rightarrow \infty} \operatorname{Re} G_{\beta}\left(\eta^{*}, \eta^{*}\right)=0 .
$$

Moreover, $d_{2} \operatorname{Im}\left(G_{\beta}\left(\eta^{*}, \eta^{*}\right)\right)-\left(\kappa d_{1} / \eta^{*}\right) \operatorname{Im}\left(\widehat{G}_{\beta}\left(\eta^{*}, \eta^{*}\right)\right)>0$ and $\operatorname{Im}\left(J_{\beta}\left(\eta^{*}, \eta^{*}\right)\right)<0$ for $\beta>0$.

Proof. First, we have $(A+i \beta)^{-1}=(A-i \beta)\left(A^{2}+\beta^{2}\right)^{-1}$. So, if $L(\beta):=\operatorname{Re}(A+i \beta)^{-1}$, then $L(\beta)=A\left(A^{2}+\beta^{2}\right)^{-1}$. Moreover, $L(\beta) \rightarrow A^{-1}$ as $\beta \rightarrow 0$ and $L(\beta) \rightarrow 0$ as $\beta \rightarrow$ $\infty$, which results in the corresponding limiting behavior for $\operatorname{Re}\left(G_{\beta}\left(\eta^{*}, \eta^{*}\right)\right)$.

To show that $\beta \mapsto\left(d_{2} \operatorname{Re}\left(G_{\beta}\left(\eta^{*}, \eta^{*}\right)\right)-\left(\kappa d_{1} / \eta^{*}\right)\right.$ $\left.\operatorname{Re}\left(\widehat{G}_{\beta}\left(\eta^{*}, \eta^{*}\right)\right)\right)$ is strictly increasing, we define $h(\beta)(x):=$ $d_{2} G_{\beta}\left(x, \eta^{*}\right)-\left(\kappa d_{1} / \eta^{*}\right) \widehat{G}_{\beta}\left(x, \eta^{*}\right)-d_{2} G\left(x, \eta^{*}\right)+\left(\kappa d_{1} / \eta^{*}\right) \widehat{G}$ $\left(x, \eta^{*}\right)$. Then (in the weak sense initially)

$$
(A+i \beta) h(\beta)=-i \beta\left(d_{2} G\left(\cdot, \eta^{*}\right)-\frac{\kappa d_{1}}{\eta^{*}} \widehat{G}\left(\cdot, \eta^{*}\right)\right) .
$$

As a result, $h(\beta) \in D(A)_{\mathrm{C}}$ and $h: \mathbb{R}^{+} \rightarrow D(A)_{\mathrm{C}}$ is differentiable with $i h(\beta)+(A+i \beta) h^{\prime}(\beta)=-i\left(d_{2} G\left(\cdot, \eta^{*}\right)-\right.$ $\left.\left(\kappa d_{1} / \eta^{*}\right) \widehat{G}\left(\cdot, \eta^{*}\right)\right)$, and therefore

$$
(A+i \beta) h^{\prime}(\beta)=-i\left(d_{2} G_{\beta}\left(\cdot, \eta^{*}\right)-\frac{\kappa d_{1}}{\eta^{*}} \widehat{G}_{\beta}\left(\cdot, \eta^{*}\right)\right) .
$$

Thus, we get

$$
\begin{aligned}
-i( & \left.d_{2}-\frac{\kappa d_{1}}{\eta^{*}}\right) \overline{h^{\prime}(\beta)\left(\eta^{*}\right)} \\
& =\int(A+i \beta)^{2} h^{\prime}(\beta) \overline{h^{\prime}(\beta)(x)} d x \\
& =\int(A+i \beta) h^{\prime}(\beta) \cdot(A+i \beta) \overline{h^{\prime}(\beta)} d x \\
& =\left\|A h^{\prime}(\beta)\right\|^{2}-\beta^{2}\left\|h^{\prime}(\beta)\right\|^{2} d x+2 i \beta\left\|A^{1 / 2} h^{\prime}(\beta)\right\|^{2} .
\end{aligned}
$$

It follows that

$$
-\left(d_{2}-\frac{\kappa d_{1}}{\eta^{*}}\right) \operatorname{Re}\left(h^{\prime}(\beta)\left(\eta^{*}\right)\right)=2 \beta\left\|A^{1 / 2} h^{\prime}(\beta)\right\|^{2}>0 .
$$

Since $\left(d_{2}-\left(\kappa d_{1} / \eta^{*}\right)\right)>0$, we have $(\partial / \partial \beta)\left(d_{2} \operatorname{Re}\left(G_{\beta}\left(\eta^{*}\right.\right.\right.$, $\left.\left.\left.\eta^{*}\right)\right)-\left(\kappa d_{1} / \eta^{*}\right) \operatorname{Re}\left(\widehat{G}_{\beta}\left(\eta^{*}, \eta^{*}\right)\right)\right)<0$ for $\beta>0$.

In order to show $\left(d_{2} \operatorname{Im}\left(G_{\beta}\left(\eta^{*}, \eta^{*}\right)\right)-\left(\kappa d_{1} / \eta^{*}\right) \operatorname{Im}\left(\widehat{G}_{\beta}\left(\eta^{*}\right.\right.\right.$, $\left.\left.\left.\eta^{*}\right)\right)\right)>0$, from (72), we have

$$
\begin{aligned}
-i \beta\left(d_{2}-\frac{\kappa d_{1}}{\eta^{*}}\right) \overline{h(\beta)\left(\eta^{*}\right)} \\
\quad=\int A(A+i \beta) h(\beta)(x) \overline{h(\beta)(x)} d x \\
\quad=\|A h(\beta)\|^{2}+i \beta\left\|A^{1 / 2} h(\beta)\right\|^{2}
\end{aligned}
$$

which implies that $-\beta\left(d_{2}-\left(\kappa d_{1} / \eta^{*}\right)\right) \operatorname{Im} h(\beta)\left(\eta^{*}\right)=$ $\|A h(\beta)\|^{2}>0$. Since $\left(d_{2}-\left(\kappa d_{1} / \eta^{*}\right)\right)>0$, we have $\operatorname{Im} h(\beta)$ $\left(\eta^{*}\right)<0$ for $\beta>0$.

Let $k(\beta)(x):=J_{\beta}\left(x, \eta^{*}\right)-J\left(x, \eta^{*}\right)$. Then we have $(\partial / \partial \beta)$ $\left(\operatorname{Re}\left(J_{\beta}\left(\eta^{*}, \eta^{*}\right)\right)\right)<0$ and $\operatorname{Im} J_{\beta}\left(\eta^{*}, \eta^{*}\right)<0$ for $\beta>0$.

Theorem 7. Under the same condition as in Theorem 4, for a unique critical point $\tau^{*}>0$, there exists a unique, purely imaginary eigenvalue $\lambda=i \beta$ of (31) with $\beta>0$.

Proof. We only need to show that the function $(u, z$, $q, \beta, \tau) \mapsto E(u, z, q, i \beta, \tau)$ has a unique zero with $\beta>0$ and $\tau>0$. This means solving the system of (31) with $\lambda=i \beta$, $u=v-\mu G\left(\cdot, \eta^{*}\right), z=p+\left(\mu / \eta^{*}\right) \widehat{G}\left(\cdot, \eta^{*}\right)$, and $q=\widehat{w}-J\left(\cdot, \eta^{*}\right)$,

$$
\begin{gathered}
(A+i \beta) v=-\mu \delta_{\eta^{*}}, \\
(A+i \beta) z=\frac{\mu}{\eta^{*}} \delta_{\eta^{*}}, \\
\left(A_{0}+i \beta\right) q=-\delta_{\eta^{*}}, \\
\frac{i \beta}{\tau^{*}}=d_{2}\left(v\left(\eta^{*}\right)+\mu G\left(\eta^{*}, \eta^{*}\right)+\gamma^{\prime}\left(\eta^{*}\right)\right) \\
+\kappa d_{1}\left(z\left(\eta^{*}\right)-\frac{\mu}{\eta^{*}} \widehat{G}\left(\eta^{*}, \eta^{*}\right)+\widehat{\gamma}^{\prime}\left(\eta^{*}\right)\right) \\
+a_{1}\left(q\left(\eta^{*}\right)+J\left(\eta^{*}, \eta^{*}\right)+\alpha^{\prime}\left(\eta^{*}\right)\right) .
\end{gathered}
$$

The real and imaginary parts of the above equation are given by

$$
\begin{aligned}
\frac{\beta}{\tau^{*}}= & d_{2} \operatorname{Im}\left(-\mu G_{\beta}\left(\eta^{*}, \eta^{*}\right)\right) \\
& +\kappa d_{1} \operatorname{Im}\left(\frac{\mu}{\eta^{*}} \widehat{G}_{\beta}\left(\eta^{*}, \eta^{*}\right)\right)-a_{1} \operatorname{Im}\left(J_{\beta}\left(\eta^{*}, \eta^{*}\right)\right) \\
0= & d_{2}\left(\operatorname{Re}\left(-\mu G_{\beta}\left(\eta^{*}, \eta^{*}\right)\right)+\mu G\left(\eta^{*}, \eta^{*}\right)+\gamma^{\prime}\left(\eta^{*}\right)\right) \\
& +\kappa d_{1}\left(\operatorname{Re}\left(\frac{\mu}{\eta^{*}} \widehat{G}_{\beta}\left(\eta^{*}, \eta^{*}\right)\right)-\frac{\mu}{\eta^{*}} \widehat{G}\left(\eta^{*}, \eta^{*}\right)\right. \\
& \left.+\alpha^{\prime}\left(\eta^{*}\right)\right) \\
& +a_{1}\left(\operatorname{Re}\left(-J_{\beta}\left(\eta^{*}, \eta^{*}\right)\right)+J\left(\eta^{*}, \eta^{*}\right)+\alpha^{\prime}\left(\eta^{*}\right)\right) .
\end{aligned}
$$


Since $d_{2} \operatorname{Im}\left(-\mu G_{\beta}\left(\eta^{*}, \eta^{*}\right)\right)+\kappa d_{1} \operatorname{Im}\left(\left(\mu / \eta^{*}\right) \widehat{G}_{\beta}\left(\eta^{*}, \eta^{*}\right)\right)-$ $a_{1} \operatorname{Im}\left(J_{\beta}\left(\eta^{*}, \eta^{*}\right)\right)>0$ by Lemma 6 , there is a critical point $\tau^{*}$, provided the existence of $\beta$. We now define

$$
\begin{gathered}
T(\beta)=d_{2}\left(\operatorname{Re}\left(-\mu G_{\beta}\left(\eta^{*}, \eta^{*}\right)\right)+\mu G\left(\eta^{*}, \eta^{*}\right)+\gamma^{\prime}\left(\eta^{*}\right)\right) \\
+\kappa d_{1}\left(\operatorname{Re}\left(\frac{\mu}{\eta^{*}} \widehat{G}_{\beta}\left(\eta^{*}, \eta^{*}\right)\right)\right. \\
\left.-\frac{\mu}{\eta^{*}} \widehat{G}\left(\eta^{*}, \eta^{*}\right)+\widehat{\gamma}^{\prime}\left(\eta^{*}\right)\right) \\
+a_{1}\left(\operatorname{Re}\left(-J_{\beta}\left(\eta^{*}, \eta^{*}\right)\right)+J\left(\eta^{*}, \eta^{*}\right)+\alpha^{\prime}\left(\eta^{*}\right)\right) .
\end{gathered}
$$

Using Lemma 6, we have $T^{\prime}(\beta)>0$ for $\beta>0$ and $T(0)=$ $d_{2} \gamma^{\prime}\left(\eta^{*}\right)+\kappa d_{1} \widehat{\gamma}^{\prime}\left(\eta^{*}\right)+a_{1} \alpha^{\prime}\left(\eta^{*}\right)=\left(d_{2}-\kappa d_{1} \sqrt{1+\mu}\right) \gamma^{\prime}\left(\eta^{*}\right)+$ $a_{1} \alpha^{\prime}\left(\eta^{*}\right)<0$ if $d_{2}>\kappa d_{1} \sqrt{1+\mu}$. Moreover,

$$
\begin{aligned}
\lim _{\beta \rightarrow \infty} T(\beta)= & d_{2}\left(\mu G\left(\eta^{*}, \eta^{*}\right)+\gamma^{\prime}\left(\eta^{*}\right)\right) \\
& +\kappa d_{1}\left(-\frac{\mu}{\eta^{*}} \widehat{G}\left(\eta^{*}, \eta^{*}\right)+\widehat{\gamma}^{\prime}\left(\eta^{*}\right)\right) \\
& +a_{1}\left(J\left(\eta^{*}, \eta^{*}\right)+\alpha^{\prime}\left(\eta^{*}\right)\right) \\
= & \left(d_{2}-\frac{\kappa d_{1}}{\eta^{*}}\right)\left(\mu G\left(\eta^{*}, \eta^{*}\right)+\gamma^{\prime}\left(\eta^{*}\right)\right) \\
& +\kappa d_{1} \widehat{\gamma}^{\prime}\left(\eta^{*}\right)+a_{1}\left(J\left(\eta^{*}, \eta^{*}\right)+\alpha^{\prime}\left(\eta^{*}\right)\right)>0
\end{aligned}
$$

for $d_{2}>\left(\kappa d_{1} / \eta^{*}\right)$ and $a_{1}>0$. Hence, there exists a unique $\beta>0$.

The following theorem summarizes the results above.

Theorem 8. Suppose that $(1 / 2)-a\left(1-s_{0}\right)<(\mu /(1+\mu))$ and $C^{\prime}\left(\gamma(\eta) ; a\left(\alpha(\eta)-s_{0}\right)\right)+\kappa \chi^{\prime \prime}(\gamma(\eta)) \widehat{\gamma}(\eta)>\kappa \sqrt{1+\mu} \chi^{\prime}(\gamma(\eta))$ for all $\eta>0$. Then (18) and (10) have at least one stationary solution $\left(u^{*}, z^{*}, q^{*}, \eta^{*}\right)$, where $u^{*}=z^{*}=q^{*}=0$, and $\left(v^{*}, p^{*}, w^{*}, \eta^{*}\right)$ where $v^{*}(x)=g\left(x, \eta^{*}\right), p^{*}(x)=\widehat{g}\left(x, \eta^{*}\right)$ and $w^{*}(x)=j\left(x, \eta^{*}\right)-s_{0}$, for all $\tau$ and for $\kappa<\kappa_{c}$, respectively, where $\kappa_{c}$ is a solution of

$$
\begin{aligned}
& C\left(\gamma(\infty) ; a\left(\alpha(\infty)-s_{0}\right)\right) \\
& \quad+\kappa_{c} \chi^{\prime}(\gamma(\infty))\left(\gamma^{\prime}(\infty)+\mu G(\infty, \infty)\right)=0 .
\end{aligned}
$$

Assume that $C^{\prime}\left(\gamma\left(\eta^{*}\right) ; a\left(\alpha\left(\eta^{*}\right)-s_{0}\right)\right)+\kappa \chi^{\prime \prime}\left(\gamma\left(\eta^{*}\right)\right) \widehat{\gamma}\left(\eta^{*}\right)>$ $\left(\kappa / \eta^{*}\right) \chi^{\prime}\left(\gamma\left(\eta^{*}\right)\right)$. Then there exists a unique $\tau^{*}$ such that the linearization $-\widetilde{A}+\tau^{*} B$ has a purely imaginary pair of eigenvalues. The point $\left(0,0,0, \eta^{*}, \tau^{*}\right)$ is then a Hopf point for (18), and there exists a $C^{0}$-curve of nontrivial periodic orbits for (18) and (10), bifurcating from $\left(0,0,0, \eta^{*}, \tau^{*}\right)$ and $\left(v^{*}, z^{*}, w^{*}, \eta^{*}, \tau^{*}\right)$, respectively.

\section{Acknowledgment}

This paper was supported by 63 Research Fund, Sungkyunkwan University, 2012.

\section{References}

[1] E. F. Keller and L. A. Segel, "Initiation of slime mold aggregation viewed as an instability," Journal of Theoretical Biology, vol. 26, pp. 399-415, 1970.

[2] K. Ikeda and M. Mimura, "Traveling wave solutions of a 3component reaction-diffusion model in smoldering combustion," Communications on Pure and Applied Analysis, vol. 11, no. 1, pp. 275-305, 2012.

[3] S. Kawaguchi, "Chemotaxis-growth under the influence of lateral inhibition in a three-component reaction-diffusion system," Nonlinearity, vol. 24, no. 4, pp. 1011-1031, 2011.

[4] M. Mimura and T. Tsujikawa, "Aggregating pattern dynamics in a chemotaxis model including growth," Physica A, vol. 230, pp. 499-543, 1996.

[5] R. Schaaf, "Stationary solutions of chemotaxis systems," Transactions of the American Mathematical Society, vol. 292, no. 2, pp. 531-556, 1985.

[6] T. Tsujikawa, "Singular limit analysis of planar equilibrium solutions to a chemotaxis model equation with growth," Methods and Applications of Analysis, vol. 3, no. 4, pp. 401-431, 1996.

[7] D. Horstmann, "From 1970 until present: the Keller-Segel model in chemotaxis and its consequences. I," Jahresbericht der Deutschen Mathematiker-Vereinigung, vol. 105, no. 3, pp. 103-165, 2003.

[8] D. Horstmann, "From 1970 until present: the Keller-Segel model in chemotaxis and its consequences. II," Jahresbericht der Deutschen Mathematiker-Vereinigung, vol. 106, no. 2, pp. 51-69, 2004.

[9] M. Ward, "Asymptotic methods for reaction-diffusion systems: past and present," Bulletin of Mathematical Biology, vol. 68, pp. 1151-1167, 2006.

[10] M. Aida, T. Tsujikawa, M. Efendiev, A. Yagi, and M. Mimura, "Lower estimate of the attractor dimension for a chemotaxis growth system," Journal of the London Mathematical Society, vol. 74, no. 2, pp. 453-474, 2006.

[11] H. Chen and X. Zhong, "Norm behaviour of solutions to a parabolic-elliptic system modelling chemotaxis in a domain of $\mathbb{R}^{3}$," Mathematical Methods in the Applied Sciences, vol. 27, no. 9, pp. 991-1006, 2004.

[12] M. Funaki, M. Mimura, and T. Tsujikawa, "Travelling front solutions arising in a chemotaxis-growth model," RIMS Kokyuroku, no. 1135, pp. 52-76, 2000.

[13] T. Nagai, T. Senba, and T. Suzuki, "Chemotactic collapse in a parabolic system of mathematical biology," Hiroshima Mathematical Journal, vol. 30, no. 3, pp. 463-497, 2000.

[14] P. Fife, "Dynamics of internal layers and diffusive interfaces," in Proceedings of the CMBS-NSF Regional Conference, vol. 53 of Applied Mathematics, SIAM, 1988.

[15] S. Kawaguchi and M. Mimura, "Collision of travelling waves in a reaction-diffusion system with global coupling effect," SIAM Journal on Applied Mathematics, vol. 59, no. 3, pp. 920-941, 1999.

[16] J. P. Keener, "A geometrical theory for spiral waves in excitable media," SIAM Journal on Applied Mathematics, vol. 46, no. 6, pp. 1039-1056, 1986.

[17] M. G. Crandall and P. H. Rabinowitz, "The Hopf bifurcation theorem in infinite dimensions," Archive for Rational Mechanics and Analysis, vol. 67, no. 1, pp. 53-72, 1977. 
[18] D. Henry, Geometric Theory of Semilinear Parabolic Equations, vol. 840 of Lecture Notes in Mathematics, Springer, Berlin, Germany, 1981.

[19] Y.-M. Lee, R. Schaaf, and R. C. Thompson, "A Hopf bifurcation in a parabolic free boundary problem," Journal of Computational and Applied Mathematics, vol. 52, no. 1-3, pp. 305-324, 1994. 


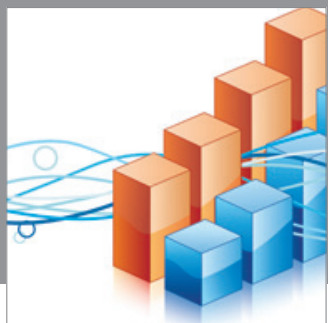

Advances in

Operations Research

mansans

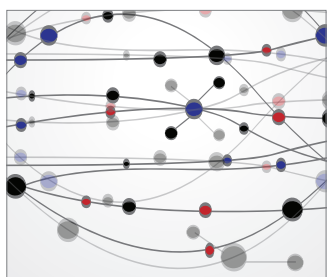

The Scientific World Journal
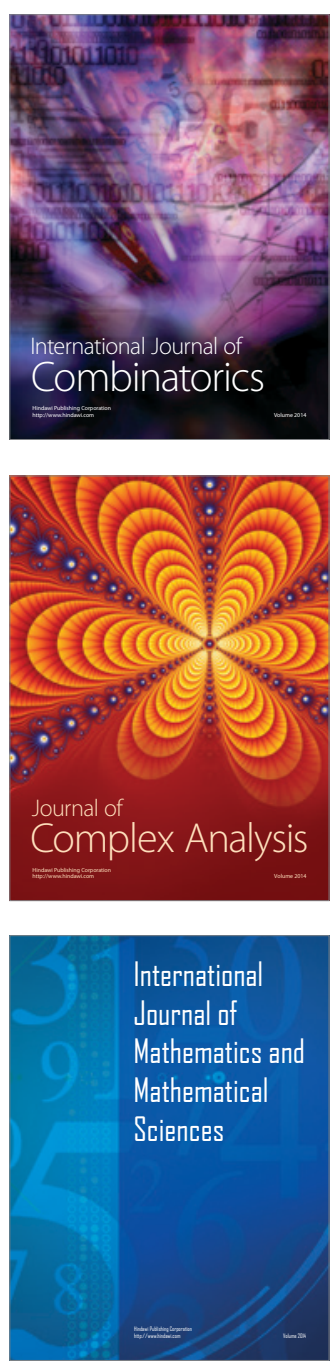
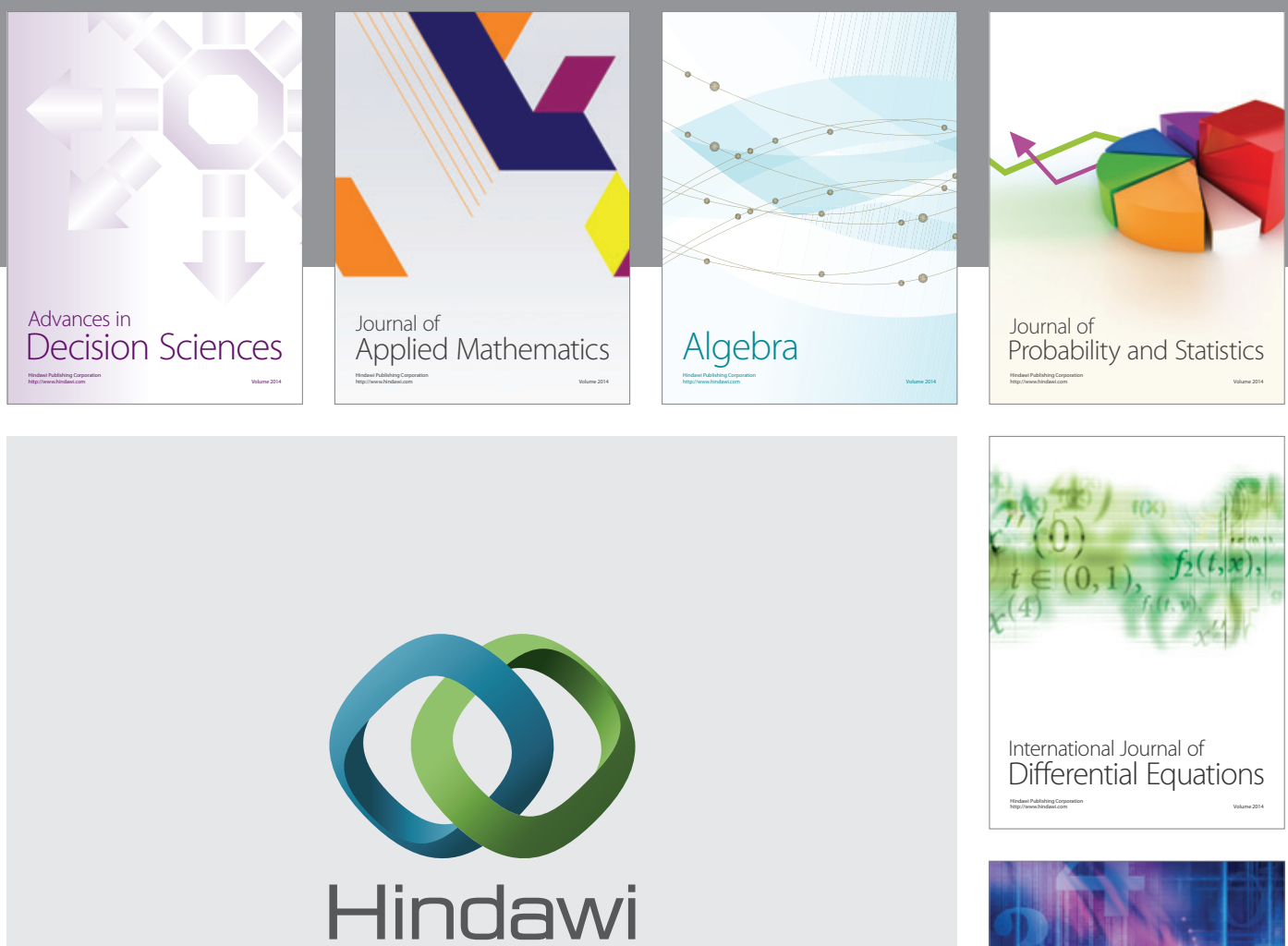

Submit your manuscripts at http://www.hindawi.com
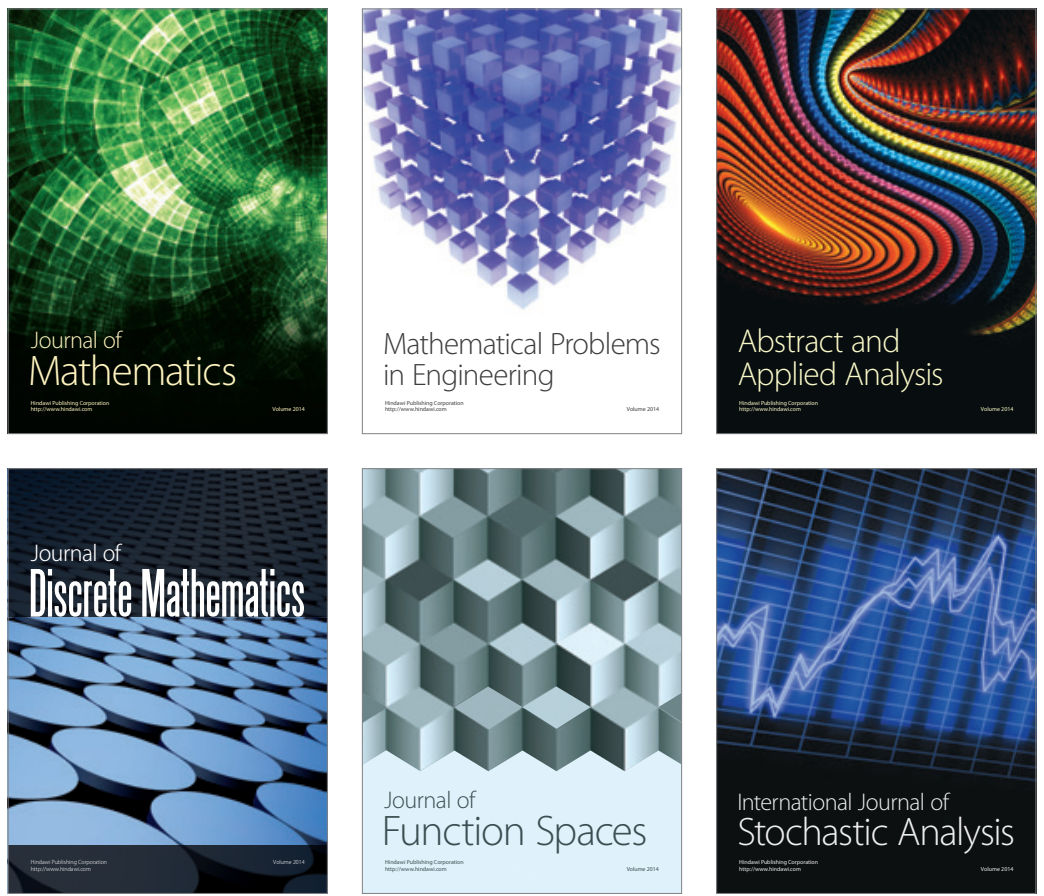

Journal of

Function Spaces

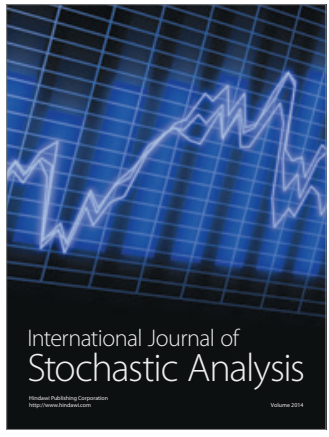

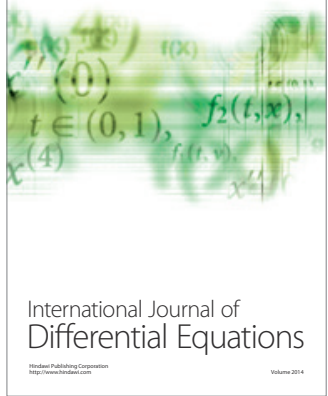
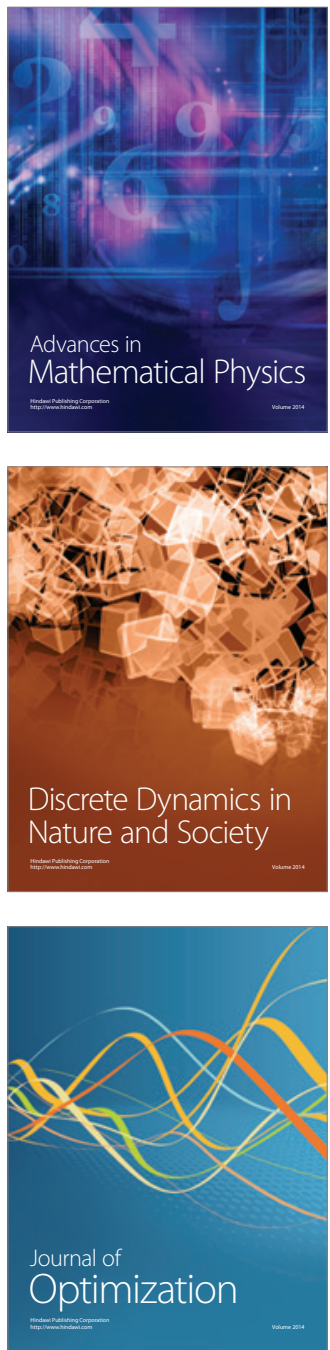